\title{
SATB2 knockdown decreases hypoxia-induced autophagy and stemness in oral squamous cell carcinoma
}

\author{
WEIJIE DONG ${ }^{1,2}$, YAWEN CHEN ${ }^{1,2}$, NAIYING QIAN ${ }^{1,2}$, GUOQI SIMA $^{1,2}$, \\ JIANMING ZHANG ${ }^{1,2}$, ZHIQIN GUO ${ }^{1,2}$ and CHANGLIN WANG ${ }^{3}$ \\ ${ }^{1}$ Department of Stomatology, The First Hospital of Jiaxing; ${ }^{2}$ Department of Stomatology, \\ First Affiliated Hospital of Jiaxing University, Jiaxing, Zhejiang 314000; ${ }^{3}$ Department of Stomatology, \\ Yancheng Hospital Affiliated to Medical School of Southeast University, Yancheng, Jiangsu 224001, P.R. China
}

Received August 18, 2019; Accepted March 13, 2020

DOI: $10.3892 / \mathrm{ol} .2020 .11589$

\begin{abstract}
Increasing evidence has suggested that special AT-rich sequence-binding protein 2 (SATB2) may be involved in the progression of numerous types of human cancer; however, the biological function of SATB2 in oral squamous cell carcinoma (OSCC) occurrence and progression remains relatively unknown. The present study aimed to investigate the potential role of SATB2 in the regulation of biological characteristics of OSSC during hypoxia. The expression of SATB2 in SCC9 cells was knocked down using small interfering RNA. Western blotting was used to determine the protein expression levels of SATB2, autophagy-related proteins microtubule-associated protein light chain (LC)3-I/II and Beclin-1, and stemness markers such as Oct-4 (POU class 5 homeobox 1), Sox-2 (SRY-box 2) and Nanog (nanog homeobox). Transmission electron microscopy and monodansylcadaverine staining were used to detect the presence of autophagosomes. Furthermore, the self-renewal capacity of cells was analyzed using colony forming assays; the cell proliferative, migratory and invasive ability were evaluated using CCK-8, wound healing and Transwell assays, respectively; and the cell cycle distribution and rate of apoptosis were detected using flow cytometry. The expression levels of SATB2, autophagy-related proteins and stemness markers were significantly increased in SCC9 cells following hypoxic treatment. Meanwhile, the genetic knockdown of SATB2 inhibited hypoxia-mediated autophagy by decreasing the expression levels of Beclin-1, and preventing the conversion of LC3-I to LC3-II and the accumulation of autophagosomes. The knockdown of SATB2 also inhibited
\end{abstract}

Correspondence to: Professor Changlin Wang, Department of Stomatology, Yancheng Hospital Affiliated to Medical School of Southeast University, 75 Juchang Road, Yancheng, Jiangsu 224001, P.R. China

E-mail: wangchanglinyk@126.com

Key words: oral squamous cell carcinoma, SATB2, autophagy, stemness, invasion the hypoxia-induced colony-forming ability and the expression of stemness markers. Functionally, it also inhibited the proliferative, migratory and invasive abilities of SCC9 cells, while inducing apoptosis and cell cycle arrest under hypoxia. In conclusion, the present study suggested that SATB2 may function as an oncogene in OSCC cells, and targeting SATB2 may be a potential therapeutic strategy for the treatment of OSCC.

\section{Introduction}

Oral squamous cell carcinoma (OSCC) is the most frequent type of head and neck squamous cell carcinoma, and it is associated with high morbidity rates and a poor prognosis worldwide $(1,2)$. OSCC is characterized as an abundance of blood and lymphatic vessels that favor tumor growth and lymphatic metastasis (3). Despite the significant progress in treatment options, including surgery, radiotherapy and chemotherapy, the five-year survival rate of patients with OSCC remains relatively low due to its high rate of recurrence (4). Therefore, it is important to understand the molecular mechanisms driving OSCC malignancy to facilitate the development of more appropriate treatment strategies to improve the prognosis and survival rates of patients with OSCC.

Special AT-rich sequence-binding protein 2 (SATB2) is a nuclear matrix attachment region binding protein that regulates gene expression and chromatin remodeling (5). It was initially identified as a gene involved in palatogenesis and craniofacial morphogenesis; however, more recent evidence has revealed that SATB2 may also serve an important role in tumor development. For example, SATB2 overexpression increased cell proliferation, stem cell-like gene expression and tumor growth in osteosarcoma (6); and SATB2 was observed to have an oncogenic role in promoting colorectal cancer tumorigenesis and progression (7). Nonetheless, the biological function of SATB2 in OSCC progression and metastasis remains poorly understood.

It is well established that the hypoxic microenvironment has a vital role in driving the invasion and metastasis of OSCC $(8,9)$. Notably, recent evidence has also suggested that hypoxia may promote stemness and autophagic properties in cancer cells, which in turn may promote the survival and 
malignancy of cancer cells during hypoxic stress that ultimately leads to poor clinical outcomes $(10,11)$. Our previous study found that SATB2 was expressed in bone marrow mesenchymal stem cells, where it served an important role in regulating the stemness and autophagy of these cells (12). Thus, the present study aimed to investigate the role of SATB2 in hypoxia-induced stemness and autophagy in OSCC, as well as its ability to regulate cellular survival, migration and invasion. The current results revealed that the expression of SATB2 was significantly increased in the OSCC cells. Silencing of SATB2 in the OSCC cells suppressed the hypoxia-induced autophagy and stemness properties, as well as aggressive behavior of OSCC cells. These findings may provide a novel target for treating the most common malignancy of the oral cavity.

\section{Materials and methods}

Ethical approval. The current study protocol was reviewed and approved by the Institutional Review Boards of The First Hospital of Jiaxing and the Yancheng Hospital Affiliated to Medical School of Southeast University.

Cell culture and transfection. The SCC9 OSCC cell line was purchased from The Cell Bank of Type Culture Collection of the Chinese Academy of Sciences. Cells were cultured in DMEM/F12 (Gibco; Thermo Fisher Scientific, Inc.) supplemented with $10 \%$ FBS (Gibco; Thermo Fisher Scientific, Inc.) and $1 \%$ penicillin/streptomycin (HyClone; GE Healthcare Life Sciences), and maintained in a humidified atmosphere at $37^{\circ} \mathrm{C}$ and $5 \% \mathrm{CO}_{2}$.

The SATB2 overexpression plasmid (pcDNA3.1-SATB2) and empty vector [negative control (NC); pcDNA3.1] were constructed and purchased from Shanghai GeneChem Co., Ltd. Small interfering RNAs (siRNAs) targeting SATB2 (si-SATB2) and the NC (si-NC) were obtained from Shanghai GenePharma Co., Ltd. The sequences of the siRNAs were as follows: si-SATB2-1, 5'-GUCAGAGAUGAGCUGAAG ATT-3'; si-SATB2-2, 5'-CCA A AC ACACCAUCAUCA AGU-3'; and si-NC, 5'-UUCUCCGAACGUGUCACGUTT-3'. Transfections of the SCC9 cells with plasmids (1 $\mu \mathrm{g} / \mathrm{well})$ and siNRAs $(50 \mathrm{nM})$ were performed using a Lipofectamine ${ }^{\circledR} 3000$ kit (Gibco; Thermo Fisher Scientific, Inc.), according to the manufacturer's protocols.

To induce hypoxic conditions, cells were transfected with siRNAs and cultured in normoxic conditions for $24 \mathrm{~h}$, prior to being exposed to a hypoxic environment of $5 \% \mathrm{CO}_{2}$, $1 \% \mathrm{O}_{2}$ and $94 \% \mathrm{~N}_{2}$ in a hypoxia incubator for $3,6,12$ and $24 \mathrm{~h}$ (Forma Scientific; Thermo Fisher Scientific, Inc.).

Monodansylcadaverine (MDC) staining. MDC staining was used to visualize autophagosomes (13). Briefly, $5 \times 10^{3}$ SCC9 cells/well were plated into 96-well plates (Corning Inc.) with DMEM/F12 medium and cultured in the incubator at $37^{\circ} \mathrm{C}$ for $6 \mathrm{~h}$. Cells were subsequently cultured with $50 \mu \mathrm{m}$ MDC (Sigma-Aldrich; Merck KGaA) for $15 \mathrm{~min}$ at $37^{\circ} \mathrm{C}$. Then, cells were fixed in $4 \%$ paraformaldehyde (Sigma-Aldrich; Merck KGaA) at room temperature for $20 \mathrm{~min}$ and washed thrice with 1X PBS (pH 7.4). The fluorescence intensity was visualized and analyzed using an Olympus IX70 inverted fluorescence microscope (magnification, x100; excitation wavelength, $340 \mathrm{~nm}$; emission wavelength, $535 \mathrm{~nm}$; Olympus Corporation). All experiments were repeated in triplicate.

Transmission electron microscopy (TEM). Following $24 \mathrm{~h}$ of hypoxic treatment, the SCC9 cells were harvested and fixed with $2.5 \%$ glutaraldehyde solution overnight at $4^{\circ} \mathrm{C}$. Then, cells were fixed using $2 \%$ osmium tetroxide for $2 \mathrm{~h}$ at room temperature, rehydrated with an increasing series of ethanol (30, 50, 70, 80, 90 and 100\%) and embedded in epoxy resin. Sections (50-70 $\mathrm{nm}$ thick) were cut using an ultramicrotome and subsequently stained with $2 \%$ uranyl acetate and $3 \%$ lead citrate for $15 \mathrm{~min}$ each at room temperature. Autophagosomes were observed under a JEM-1400Plus transmission electron microscope (magnification, x6,000; JEOL, Ltd.).

Colony forming assay. The colony forming assay was performed as previously described (12). Briefly, single cell suspensions of $1 \times 10^{3}$ cells/well were plated into 6-well plates (Corning Inc.) and cultured in complete DMEM/F12 at $37^{\circ} \mathrm{C}$ and $1 \% \mathrm{CO}_{2}$ for 12 days; the medium was changed every 3 days. Upon incubation, the visible colonies were fixed in $4 \%$ paraformaldehyde (Sigma-Aldrich) at room temperature for $20 \mathrm{~min}$ and subsequently stained with a $0.1 \%$ crystal violet solution (Sigma-Aldrich; Merck KGaA) for $30 \mathrm{~min}$ at room temperature. The stained cells were visualized under an inverted microscope (magnification, $x 40$; Olympus Corporation), and the number of colonies with $\geq 50$ cells in each well were counted.

Wound healing assay. The migratory ability of cells was analyzed using a wound healing assay. Briefly, when the monolayer of cells reached $100 \%$ confluence in 6-well plates, a $20-\mu 1$ sterile pipette tip was used to create the wound. Cells were washed with 1X PBS three times to remove the detached and damaged cells. Then, the cells were incubated under hypoxic conditions for $24 \mathrm{~h}$ with serum-free DMEM/F12. At 0 and 24-h incubation, the cells were imaged using an inverted light microscope (magnification, x100; Olympus Corporation), and the wound closure area was used to calculate the migratory ability of cells using ImageJ software (version 1.48, National Institutes of Health). Each experiment was performed in triplicate.

Cell invasion assay. The invasive ability of cells was determined using a Transwell assay. A total of $5 \times 10^{4}$ SCC9 cells were resuspended in $200 \mu \mathrm{l}$ serum-free DMEM/F12 and plated in the upper chambers of Transwell plates (pore size, 8- $\mu \mathrm{m}$; Costar; Corning Inc.) which pre-coated with $25 \mu \mathrm{l}$ Matrigel (BD Biosciences) at $37^{\circ} \mathrm{C}$ for $30 \mathrm{~min}$. A total of $500 \mu \mathrm{l}$ complete DMEM/F12 was plated in the lower chambers. Following incubation at $37^{\circ} \mathrm{C}$ in the hypoxia incubator for $24 \mathrm{~h}$, the cells were fixed in $4 \%$ PFA for $20 \mathrm{~min}$ at room temperature and subsequently stained with $0.1 \%$ crystal violet (Sigma-Aldrich; Merck KGaA) for $30 \mathrm{~min}$ at room temperature. The non-invasive cells remaining in the upper chambers were removed with a swab, and the invasive cells present on the lower surface were counted in 10 randomly selected fields using an inverted microscope (magnification, x100; Olympus Corporation). 
Cell proliferation assay. The proliferative ability of SCC9 cells was evaluated by CCK- 8 assay (Beyotime Institute of Biotechnology), according to the manufacturer's protocols. Briefly, $1 \times 10^{3}$ SCC9 cells/well with or without siRNA transfection were seeded into 96-well plates. At 24, 48 and $72 \mathrm{~h}$, $10 \mu \mathrm{l} \mathrm{CCK}-8$ reagent was added into each well, and the plates were incubated at $37^{\circ} \mathrm{C}$ for additional $2 \mathrm{~h}$. The absorbance was measured at $450 \mathrm{~nm}$ using a microplate spectrophotometer (Thermo Fisher Scientific Inc.). Each experiment was performed in triplicate.

Cell cycle assay. Following incubation under hypoxia for $24 \mathrm{~h}$, the SCC9 cells were harvested, washed with cold PBS and fixed in $70 \%$ ice-cold ethanol overnight at $-20^{\circ} \mathrm{C}$. The cells were subsequently stained with $50 \mu \mathrm{g} / \mathrm{ml}$ propidium iodide (PI) and $20 \mu \mathrm{g} / \mathrm{ml}$ RNase A (BD Biosciences) at $4^{\circ} \mathrm{C}$ for $30 \mathrm{~min}$. After incubation, the cells were subjected to DNA content analysis using a BD FACSCalibur flow cytometer (LSR II; BD Biosciences), and the data were analyzed using CellQuest $^{\mathrm{TM}}$ Pro version 5.2 software (BD Biosciences).

Cell apoptosis assay. Annexin V-fluorescein isothiocyanate (FITC)/propidium iodide (PI) Apoptosis Detection kit (BD Biosciences) was performed to detect apoptosis according to the manufacturer's instructions. Briefly, after washing with PBS, SCC9 cells were collected by trypsinization with $0.25 \%$ trypsin solution without EDTA and resuspended in $500 \mu \mathrm{l} 1 \mathrm{X}$ binding buffer from the kit. Then, $5 \mu \mathrm{l}$ Annexin V-FITC and $5 \mu \mathrm{l}$ PI were added for $15 \mathrm{~min}$ at room temperature in the dark. Cell apoptosis was subsequently detected using a BD FACSCalibur flow cytometer (BD Biosciences), and data were analyzed using FlowJo version 9.0 software (FlowJo LLC). Each experiment was performed in triplicate.

Western blotting. Total protein was extracted from SCC9 cells using RIPA lysis buffer (Beyotime Institute of Biotechnology), containing a protease inhibitor and phenylmethylsulfonyl fluoride. Following centrifugation of the lysates at $12,000 \mathrm{x} \mathrm{g}$ for 15 min at $4^{\circ} \mathrm{C}$, the supernatants were collected, and total protein was quantified using a bicinchoninic assay kit (Beyotime Institute of Biotechnology). Equal amounts of protein from each sample (40 $\mu \mathrm{g} /$ well) were separated by $10 \%$ SDS-PAGE and subsequently transferred onto polyvinylidene fluoride membranes (EMD Millipore). Membranes were blocked with 5\% skimmed milk (Sigma-Aldrich; Merck KGaA) in TBS-0.05\% Tween-20 (TBST) buffer (Sangon Biotech Co., Ltd.) at room temperature for $2 \mathrm{~h}$ and then incubated with the following primary antibodies $(1: 1,000)$ at $4^{\circ} \mathrm{C}$ overnight: Anti-hypoxia-inducible factor $1-\alpha$ (HIF-1 $\alpha$; cat. no. 3716; Cell Signaling Technology, Inc.), anti-SATB2 (cat. no. ab34735; Abcam), anti-microtubule-associated protein light chain 3-II/I (LC3-II/I; cat. no. 2775; Cell Signaling Technology, Inc.), anti-Beclin-1 (cat. no. 3495; Cell Signaling Technology, Inc.), anti-Oct-4 (cat. no. ab137427; Abcam), anti-Sox-2 (cat. no. ab171380; Abcam), anti-Nanog (cat. no. ab70482; Abcam) and anti- $\beta$-actin (cat. no. 3700; Cell Signaling Technology, Inc.). Following the primary antibody incubation, the membranes were washed three times with TBST and subsequently incubated with horseradish peroxidase-conjugated secondary antibodies (cat. nos. 7074 and 7076; Cell Signaling Technology, Inc.) for $1 \mathrm{~h}$ at room temperature. Protein bands were visualized using an enhanced chemiluminescence detection kit (PerkinElmer, Inc.) and a Bio-Rad gel image analysis system (Bio-Rad Laboratories, Inc.). Protein expression was quantified using ImageJ version $1.5 \mathrm{~b}$ software (National Institutes of Health) and normalized to $\beta$-actin as the loading control.

Statistical analysis. Statistical analysis was performed using GraphPad Prism 5 software (GraphPad Software, Inc.), and all data were presented as the mean \pm SD from $\geq 3$ replicates for each experiment. Statistical differences amongst groups were determined using one-way ANOVA, followed by Dunnett's test for multiple comparisons. $\mathrm{P}<0.05$ was considered to indicate a statistically significant difference.

\section{Results}

Hypoxia induces the expression of SATB2 in SCC9 cells. Following the exposure of SCC9 cells to hypoxia for 3, 6, 12 or $24 \mathrm{~h}$, western blotting revealed that HIF-1 $\alpha$ protein expression levels began to increase significantly within $3 \mathrm{~h}$, peaked at $12 \mathrm{~h}$ and then decayed at $24 \mathrm{~h}$. A similar pattern was observed with SATB2 expression levels, indicating that SATB2 may serve a critical role in OSCC progression under hypoxia (Fig. 1A). To further investigate the role of SATB2 in OSCC progression, the expression of SATB2 was inhibited by transfecting si-SATB2 into SCC9 cells under hypoxic conditions. The western blotting results revealed that SATB2 expression was knocked down by $\sim 45 \%$ in si-SATB2-1-transfected cells and by $\sim 80 \%$ in si-SATB2-2-transfected cells, whereas HIF-1 $\alpha$ protein levels remained unaltered after SATB2 silencing (Fig. 1B). Therefore, the si-SATB2-2 sequence was used for subsequent experiments.

SATB2 knockdown suppresses hypoxia-induced autophagy in SCC 9 cells. To investigate the potential role of SATB2 in hypoxia-induced autophagy, the expression levels of the autophagy markers LC3-II/I and Beclin-1 were determined in hypoxia-treated SCC9 cells. Western blotting revealed that the conversion of endogenous LC3-I to LC3-II and the expression levels of Beclin-1 were significantly increased in SCC9 cells exposed to hypoxia compared with cells under normoxia (Fig. 2A). By contrast, the genetic knockdown of SATB2 significantly decreased the LC3-II/LC3-I ratio and expression levels of Beclin-1 in SCC9 cells compared with the control group under hypoxia (Fig. 2B).

To further identify the effect of SATB2 on autophagy, TEM and MDC staining (a marker for autophagic vacuoles) were also used. TEM observed that a greater number of autophagosomes accumulated in hypoxic control cells compared with si-SATB2-transfected cells (Fig. 2C and D). Similarly, MDC staining demonstrated that the relative fluorescence intensity of si-SATB2-transfected cells was significantly decreased when compared with hypoxic control group (Fig. 2E and F). Together, these observations indicated that SATB2 may regulate autophagy in SCC9 cells under hypoxic conditions.

SATB2 knockdown suppresses hypoxia-induced stemness of SCC 9 cells. To investigate the effect of SATB2 on the 

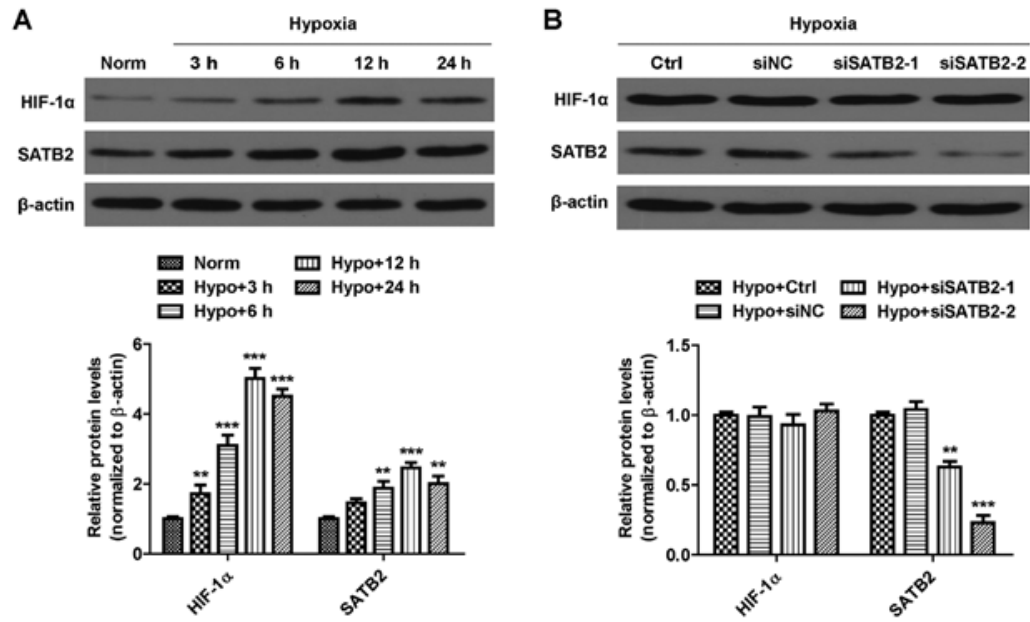

Figure 1. Expression levels of HIF-1 $\alpha$ and SATB2 in SCC9 cells. (A) The relative protein expression levels of HIF-1 $\alpha$ and SATB2 were examined by western blot analysis in SCC9 cells exposed to normoxic or hypoxic conditions for the indicated times. ${ }^{* *} \mathrm{P}<0.01,{ }^{* * * *} \mathrm{P}<0.001$ vs. normoxia group. (B) The relative protein expression levels of HIF-1 $\alpha$ and SATB2 were detected by western blot analysis in SCC9 cells transfected with si-NC, siSATB2-1 and si-SATB2-2, and cultured under hypoxic condition for $24 \mathrm{~h}$. Protein levels were normalized by comparison with $\beta$-actin levels. The results are presented as the mean \pm SD of three independent experiments. ${ }^{* *} \mathrm{P}<0.01,{ }^{* * *} \mathrm{P}<0.001$ vs. hypoxia control group. HIF-1 $\alpha$, hypoxia-inducible factor $1-\alpha$; SATB2, special AT-rich sequence-binding protein 2; si, small interfering; NC, negative control; norm, normoxia; hypo, hypoxic; ctrl, control.

A

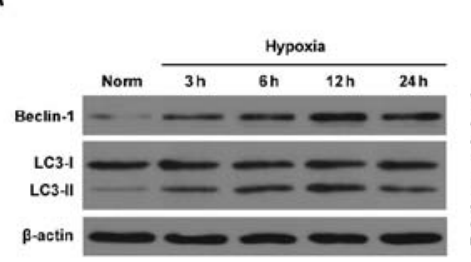

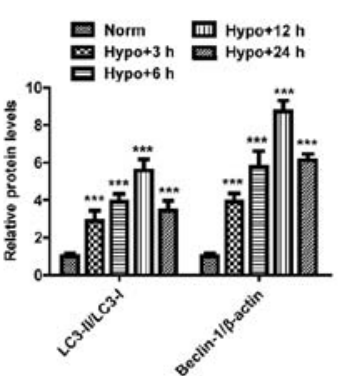

C

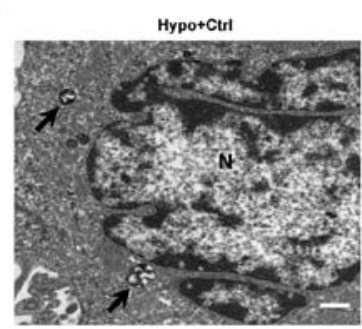

E

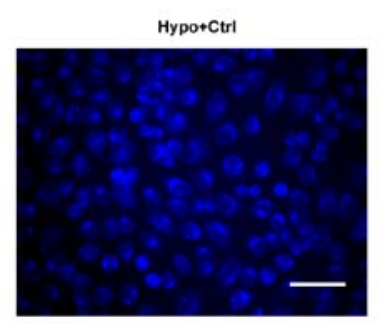

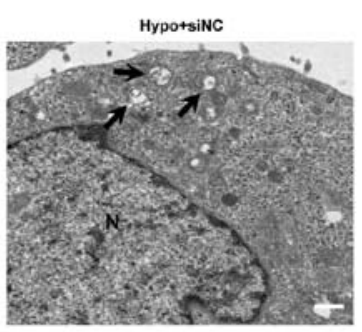
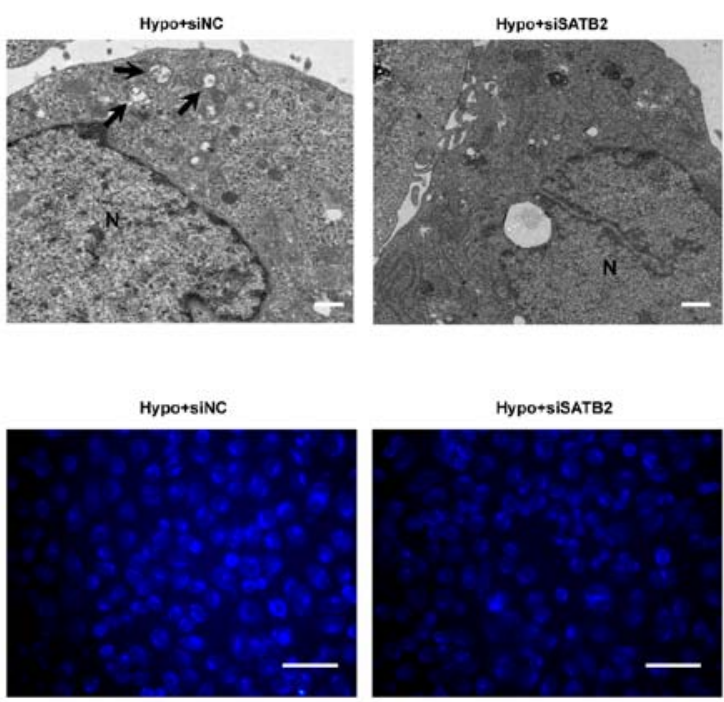

B
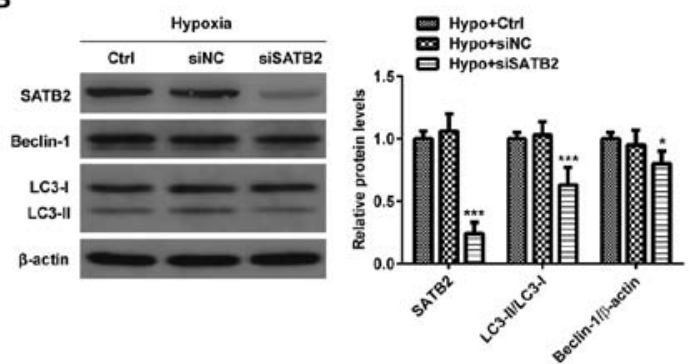

D

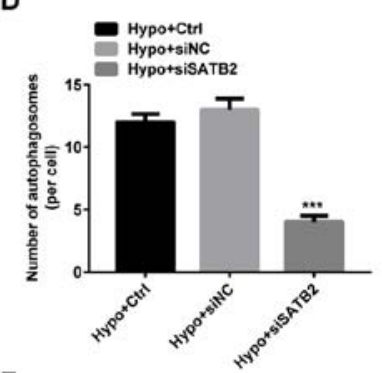

$\mathbf{F}$

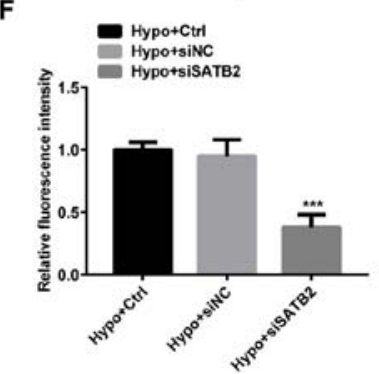

Figure 2. SATB2 knockdown suppresses hypoxia-induced autophagy in SCC9 cells. (A) The relative protein expression levels of LC3-I, LC3-II and Beclin-1 were examined by western blot analysis in SCC9 cells exposed to normoxic or hypoxic conditions for the indicated times. Protein levels were normalized by comparison with $\beta$-actin levels. ${ }^{* * * *} \mathrm{P}<0.001$ vs. normoxia group. (B) The relative protein expression levels of SATB2, LC3-I, LC3-II and Beclin-1 were detected by western blot analysis in SCC9 cells transfected with either si-NC or si-SATB2-2, and cultured under hypoxic conditions for $24 \mathrm{~h}$. Protein levels were normalized by comparison with $\beta$-actin levels. "P<0.05, ${ }^{* * *} \mathrm{P}<0.001$ vs. hypoxia control group. (C) Representative TEM images of autophagosomes in SCC9 cells transfected with either si-NC or si-SATB2-2 and cultured under hypoxic conditions for $24 \mathrm{~h}$. Black arrows indicate autophagy vacuoles. N indicates nuclear. Scale bar=1 $\mu \mathrm{m}$. (D) The number of autophagosomes per cross-sectioned cell was counted (20 cells per group) ${ }^{* * * *} \mathrm{P}<0.001$ vs. hypoxia control group. (E) Representative fluorescent images of MDC staining in SCC9 cells transfected with either si-NC or si-SATB2-2 and cultured under hypoxic conditions for $24 \mathrm{~h}$. Scale bar=100 $\mu \mathrm{m}$. (F) ImageJ was used to calculate the relative fluorescence intensity of MDC. The results are presented as the mean \pm SD of three independent experiments. ${ }^{* * *} \mathrm{P}<0.001$ vs. hypoxia control group. SATB2, special AT-rich sequence-binding protein 2; LC3, microtubule-associated protein light chain 3; si, small interfering; NC, negative control; norm, normoxia; hypo, hypoxic; ctrl, control; TEM, transmission electron microscopy; MDC, monodansylcadaverine. 
A
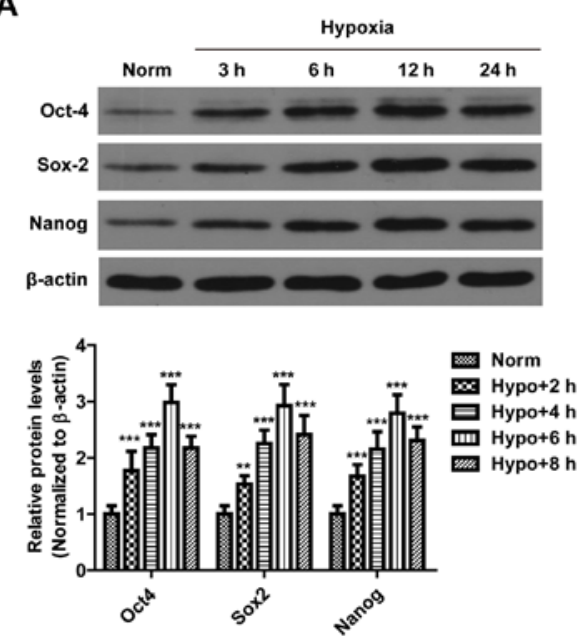

C

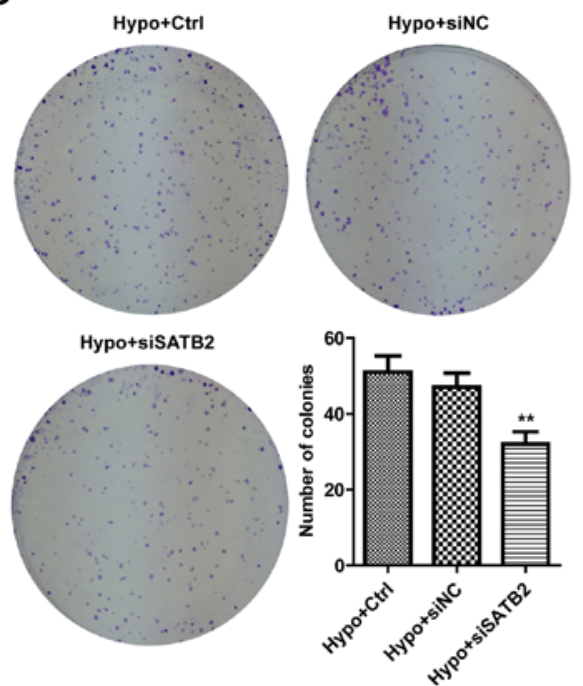

B
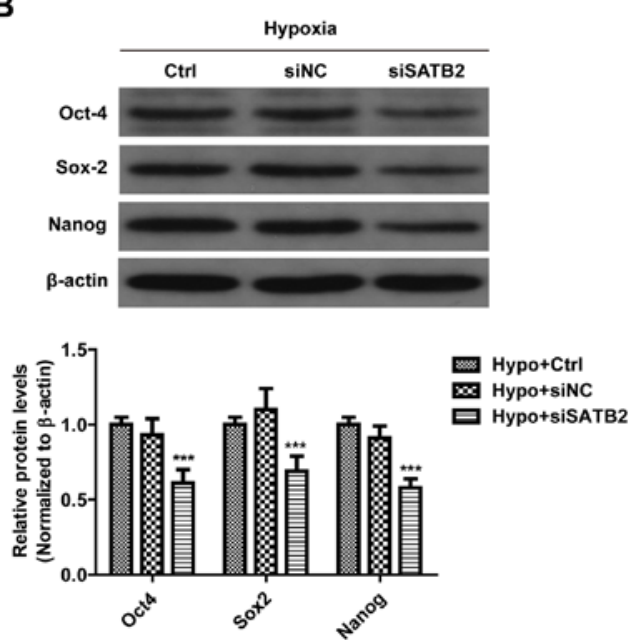

D

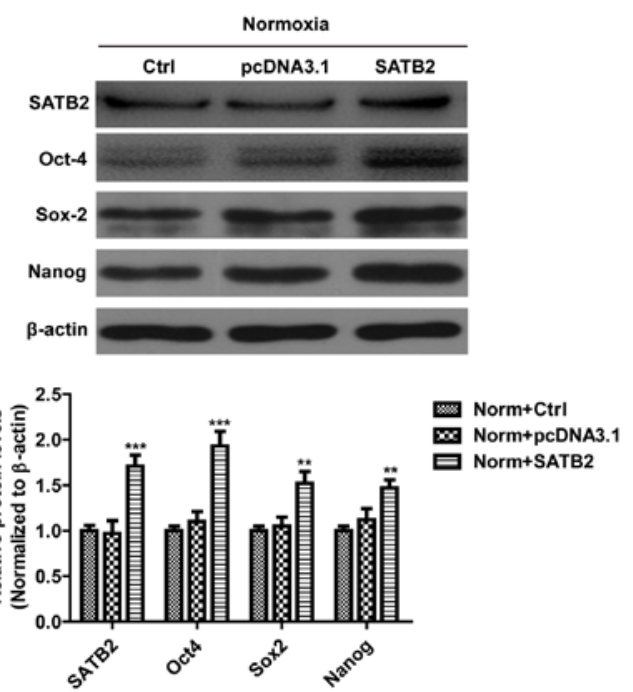

Figure 3. SATB2 knockdown suppresses hypoxia-induced stemness of SCC9 cells. (A) The relative protein expression levels of Oct-4, Sox-2 and Naong were examined by western blot analysis in SCC9 cells exposed to normoxic or hypoxic conditions for the indicated times. Protein levels were normalized by comparison with $\beta$-actin levels. ${ }^{* *} \mathrm{P}<0.01,{ }^{* * *} \mathrm{P}<0.001$ vs. normoxia group. (B) Relative protein expression levels of Oct- 4 , Sox-2 and Nanog were detected by western blot analysis in SCC9 cells transfected with either si-NC or si-SATB2-2 and cultured under hypoxic conditions for 24 h. Protein levels were normalized by comparison with $\beta$-actin levels. ${ }^{* * *} \mathrm{P}<0.001$ vs. hypoxia control group. (C) Images of colonies stained with crystal violet, and quantification of the number of colonies formed in SCC9 cells transfected with either si-NC or si-SATB2-2 and cultured under hypoxic conditions for 12 days. ${ }^{* * *} \mathrm{P}<0.01$ vs. hypoxia control group. (D) The relative protein expression levels of Oct-4, Sox-2 and Nanog were detected by western blot analysis in SCC 9 cells transfected with either empty (pcDNA3.1) or SATB2-overexpression plasmids and cultured under normoxic conditions. ${ }^{* *} \mathrm{P}<0.01,{ }^{* * *} \mathrm{P}<0.001$ vs. normoxia group. The results are presented as the mean \pm SD of three independent experiments. SATB2, special AT-rich sequence-binding protein 2; si, small interfering; NC, negative control; norm, normoxia; hypo, hypoxic; ctrl, control.

hypoxia-mediated stemness phenotype, the expression levels of the stemness-related markers Oct-4, Sox-2 and Nanog were evaluated in SCC9 cells during hypoxic and normoxic conditions. The protein expression levels of Oct-4, Sox-2 and Nanog in hypoxic cells were significantly increased compared with those in normoxic cells (Fig. 3A). By contrast, knocking down SATB2 decreased the expression levels of Oct-4, Sox-2 and Nanog, as well as the colony-forming ratio of SCC9 cells (Fig. 3B and C). Moreover, following the transfection of empty and SATB2-overexpression plasmids into SCC9 cells under normoxia, the western blotting results revealed that SATB2 overexpression increased the protein expression levels of stemness-related markers (Fig. 3D). Taken together, the results indicated that the genetic silencing of SATB2 may inhibit the hypoxia-mediated stemness phenotype in SCC9 cells.
SATB2 knockdown inhibits the proliferation while promoting the apoptosis of hypoxia-exposed SCC 9 cells. To verify the effect of SATB2 on the proliferative ability of SCC9 cells, both CCK-8 and cell cycle assays were performed. The results from the CCK- 8 assay demonstrated that the knockdown of SATB2 significantly suppressed the proliferative ability of SCC9 cells under hypoxia (Fig. 4A). Furthermore, as anticipated, cells transfected with si-SATB2 induced G0/G1 cell cycle arrest, which manifested through a markedly higher percentage of cells in the G0/G1 phase and a lower percentage of cells in the S phase (Fig. 4B and D). Subsequently, Annexin V-FITC/PI assays were used to investigate the effects of SATB2 knockdown on SCC9 cell apoptosis. The si-SATB2-transfected group exhibited a significantly increased number of Annexin V-positive cells 
A

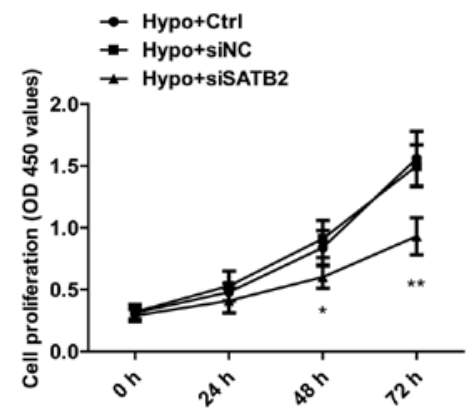

B

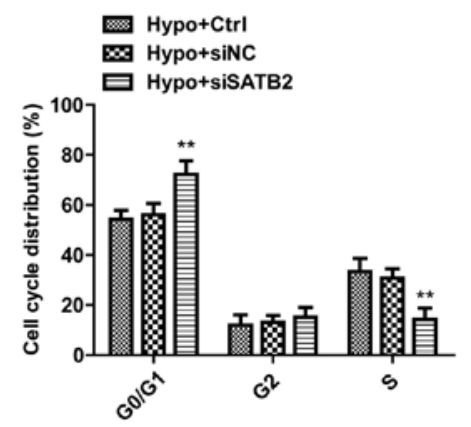

C

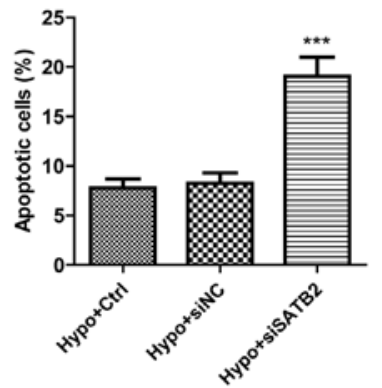

Hypo+siSATB2
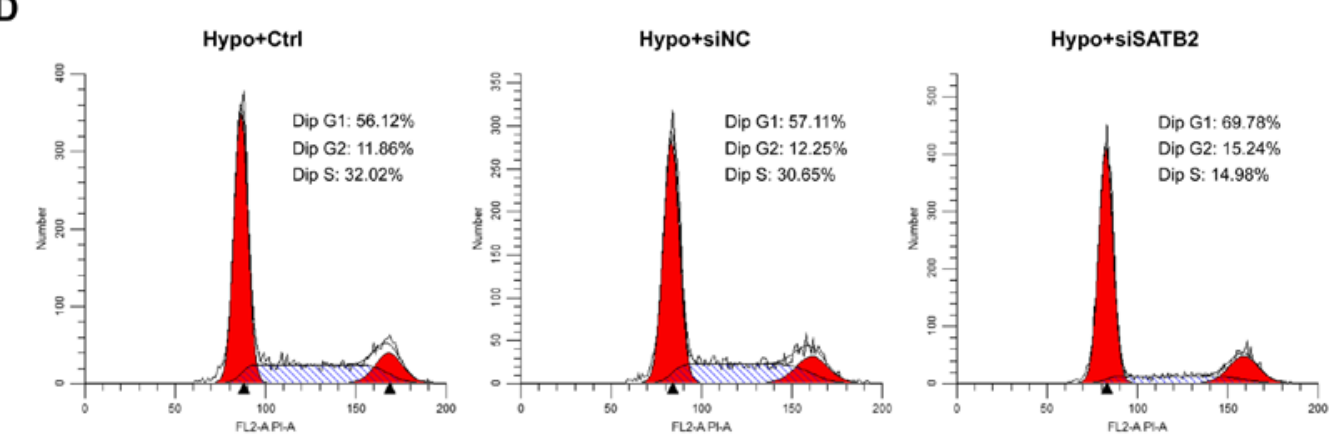

E
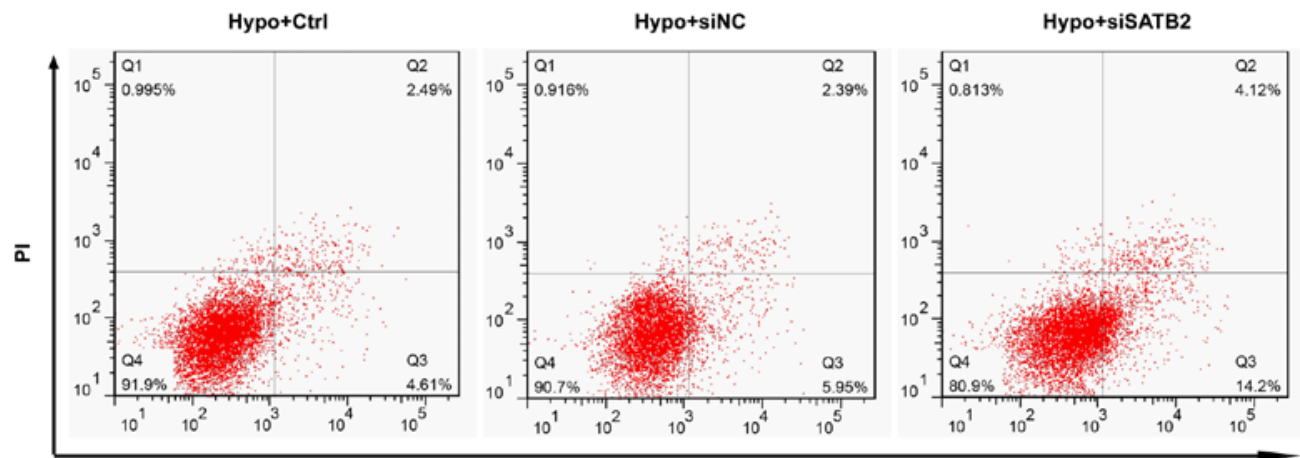

Annexin-V FITC

Figure 4. SATB2 knockdown inhibits the proliferation while promoting the apoptosis of hypoxia-exposed SCC9 cells. (A) The cell proliferation capacity was determined by CCK-8 assay in SCC9 cells transfected with either si-NC or si-SATB2-2, and cultured under hypoxic conditions for 24,48 and $72 \mathrm{~h}$ (B and D) Cell cycle was detected by flow cytometry with PI staining in SCC9 cells transfected with either si-NC or si-SATB2-2 and cultured under hypoxic conditions for 24 h. (C and E) Cell apoptosis was detected by flow cytometry with Annexin V-FITC and PI double staining in SCC9 cells transfected with either si-NC or si-SATB2-2 and cultured under hypoxic conditions for $24 \mathrm{~h}$. The results are presented as the mean \pm SD of three independent experiments. ${ }^{*} \mathrm{P}<0.05,{ }^{* *} \mathrm{P}<0.01,{ }^{* * *} \mathrm{P}<0.001$ vs. hypoxia control group. SATB2, special AT-rich sequence-binding protein 2; si, small interfering; NC, negative control; hypo, hypoxic; ctrl, control; PI, propidium iodide.

compared with the control or si-NC group under hypoxic conditions (Fig. 4C and E). Overall, these findings indicated that SATB2 knockdown may inhibit cell growth and induce cell apoptosis in hypoxic SCC9 cells.

SATB2 knockdown inhibits the migration and invasion of hypoxia-exposed SCC 9 cells. To determine the role of SATB2 in SCC9 cell migration under hypoxic conditions, wound healing assays were used to measure the cell migratory ability. In SATB2 knockdown cells, the migration rate was significantly reduced (Fig. 5A and B). The effect of SATB2 on cell invasion was detected using Transwell assays. Compared with the hypoxic control group, the number of invasive cells in the si-SATB2 group was significantly decreased (Fig. 5C and D).
These data suggested that SATB2 silencing may inhibit migratory and invasive processes in SCC9 cells under hypoxic conditions.

\section{Discussion}

An increasing number of studies have observed that SATB2 is aberrantly expressed in numerous types of malignant tumor, including OSCC $(6,7,14-16)$. For example, Chung et al (16) previously reported that SATB2 was preferentially expressed in advanced-stage primary OSCC, and that the knockdown of SATB2 re-sensitized OSCC cells to chemotherapy-induced apoptosis. However, the role of SATB2 in regulating autophagic and stemness properties 

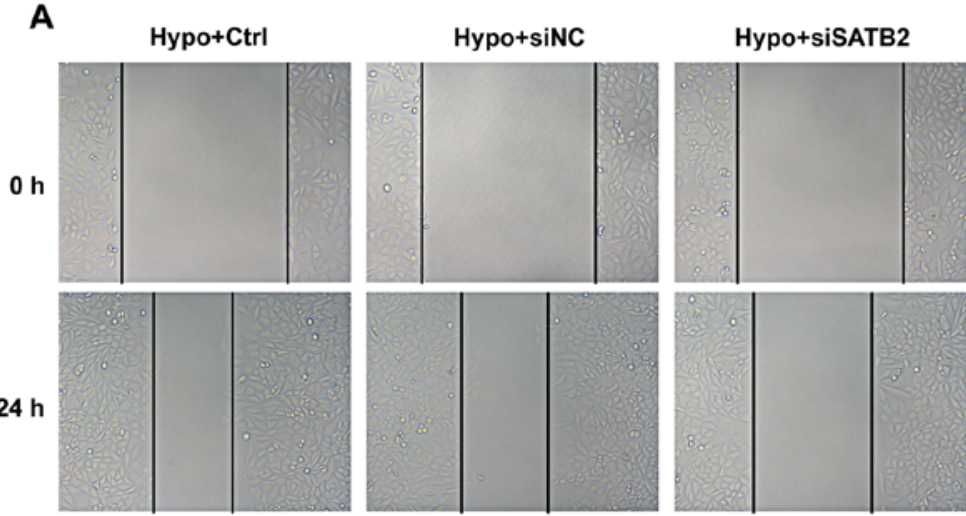

C
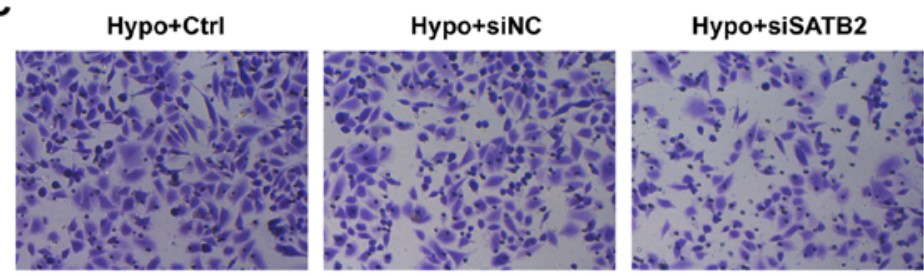

B

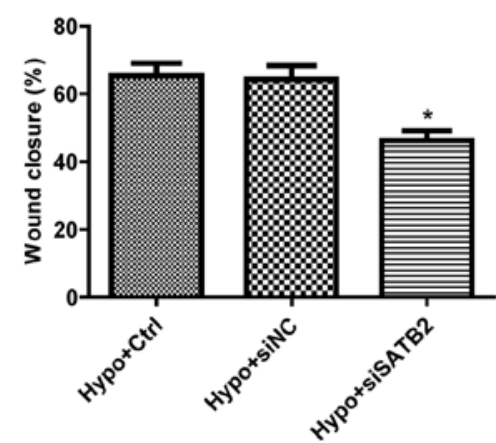

D

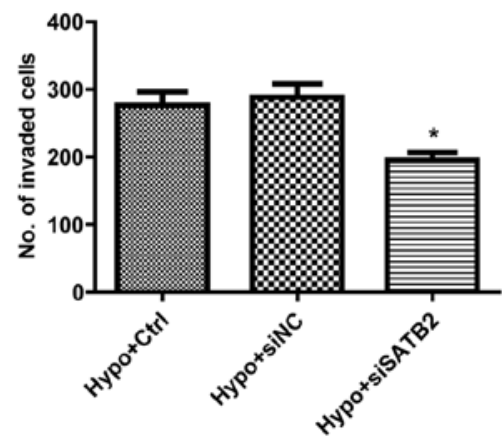

Figure 5. SATB2 knockdown inhibits migration and invasion of hypoxia-exposed SCC9 cells. (A and B) Cell migration was detected by wound healing assay in SCC9 cells transfected with either si-NC or si-SATB2-2 and cultured under hypoxic conditions for $24 \mathrm{~h}$. (C and D) Cell invasion was detected by Transwell invasion assay in SCC9 cells transfected with either si-NC or si-SATB2-2 and cultured under hypoxic conditions for $24 \mathrm{~h}$. The results are presented as the mean \pm SD of three independent experiments. ${ }^{*} \mathrm{P}<0.05$ vs. hypoxia control group.

of cancer cells remains relatively unclear, and, to the best of our knowledge, it has yet to be investigated in OSCC cells. In the present study, the expression levels of SATB2 were significantly increased in SCC9 cells under hypoxic conditions, whereas the genetic silencing of SATB2 did not regulate the expression of HIF-1 $\alpha$, suggesting that SATB2 is one of the downstream molecules of HIF-1 $\alpha$. Moreover, SATB2 knockdown suppressed the hypoxia-induced autophagy and stemness properties of SCC9 cells, and consequently suppressed their proliferative, migratory and invasive ability, while stimulating cell cycle arrest and apoptosis in SCC9 cells under hypoxia. These findings suggested that SATB2 may be a novel target for the treatment of OSCC.

Apoptosis and autophagy are two crucial processes that maintain cellular homeostasis in physiological and pathological conditions, in which crosstalk between the two pathways can occur. Previously, hypoxia-induced autophagy was demonstrated to promote tumor cell survival by eliminating potentially toxic macromolecules and damaged organelles $(17,18)$. Moreover, several previous studies in OSCC have reported that the inhibition of autophagy enhances apoptotic cell death, suggesting that a combination treatment of anticancer drugs and autophagy inhibitors may be an effective strategy for OSCC treatment (19-21). In the present study, hypoxia-induced classic hallmarks of autophagy in SCC9 cells were observed, including accumulation of autophagosomes, conversion of LC3-I to LC3-II and increased expression levels of Beclin-1. Moreover, the knockdown of SATB2 using RNA interference was found to suppress hypoxia-induced autophagy and promote apoptosis in SCC9 cells. Overall, our findings indicate that SATB2 may inhibit cellular apoptosis partially through promoting autophagy in OSCC.

It has been suggested that the acquisition of stem-like properties by cancer cells markedly contributes to cancer recurrence and poor prognosis $(22,23)$. With this in mind, it has been previously reported by Yu et al (14) that the overexpression of SATB2 in human pancreatic normal ductal epithelial cells increased the expression levels of the stem cell markers CD44, CD24 and CD133, and the transcription factors Oct-4, Sox-2 and Nanog. However, Li et al (24) found that SATB2 directly bound to the regulatory elements of stem cell markers such as CD133, CD44, meis homeobox 2 and axin 2, and consequently inhibited the progression of colorectal cancer by negatively regulating the stemness of colorectal cancer cells. Therefore, the roles of SATB2 on the biological function of cancer cells are dependent on the tumor cell line. Based on loss-of-function experiments, the results of the current study were consistent with those found by Yu et al (14); the present findings demonstrated that the knockdown of SATB2 inhibited the expression of the hypoxia-induced stemness factors Oct-4, Sox-2 and Nanog, in addition to preventing colony formation, which suggested that the stemness phenotype was inhibited following SATB2 knockdown. Due to the strong association between epithelial-to-mesenchymal transition (EMT) and stemness in OSCC cells (25), further studies are required to investigate the effect of SATB2 on EMT processes.

In the present study, SATB2 knockdown was observed to inhibit cell proliferation and facilitate cell cycle arrest, demonstrating an increased G0/G1 phase fraction and a decreased 
$\mathrm{S}$ phase fraction in SCC9 cells that were exposed to hypoxia. Considering that cell cycle progression is one of the main causes of the proliferation of tumor cells, multiple studies have suggested that cell cycle-associated genes may be primary targets for cancer treatment $(26,27)$. In addition, a significant decrease in both the cellular migratory and invasive ability was observed following SATB2 gene silencing. Thus, to the best of our knowledge, this is the first study to suggest that SATB2 may exert multiple biological functions in OSCC.

In conclusion, the present study demonstrated that SATB2 may serve as an oncogene in SCC9 cells by accelerating autophagy and stemness processes. The silencing of SATB2 gene expression inhibited the proliferative, migratory and invasive ability of OSCC cells, which are all important processes that are required for OSCC progression. In future studies, OSCC animal models should be used to further investigate the function of SATB2 and its relevance in OSCC.

\section{Acknowledgements}

Not applicable.

\section{Funding}

The present study was supported by grants from the Science and Technology Plan Project of Jiaxing, Zhejiang, China (grant no. 2016BY28004) and the Medical and Health Science and Technology Project of Zhejiang Province, China (grant no. 2017196633).

\section{Availability of data and materials}

The datasets used and/or analyzed during the current study are available from the corresponding author on reasonable request.

\section{Authors' contributions}

WD and CW designed the study and wrote the initial draft of the article. YC, NQ, GS, JZ and ZG contributed to analysis and interpretation of data and assisted in the preparation of the article. WD, YC, NQ and GS performed the experiments. All authors contributed to data collection and interpretation, and critically reviewed the article. All authors read and approved the final version of the manuscript and agree to be accountable for all aspects of the work in ensuring that questions related to the accuracy or integrity of any part of the work are appropriately investigated and resolved.

\section{Ethics approval and consent to participate}

Not applicable.

\section{Patient consent for publication}

Not applicable.

\section{Competing interests}

The authors declare that they have no competing interests.

\section{References}

1. Torre LA, Bray F, Siegel RL, Ferlay J, Lortet-Tieulent J and Jemal A: Global cancer statistics, 2012. CA Cancer J Clin 65: 87-108, 2015

2. Krishna Rao SV, Mejia G, Roberts-Thomson K and Logan R: Epidemiology of oral cancer in Asia in the past decade-an update (2000-2012). Asian Pac J Cancer Prev 14: 5567-5577, 2013.

3. Ausoni S, Boscolo-Rizzo P, Singh B, Da Mosto MC, Spinato G, Tirelli G, Spinato R and Azzarello G: Targeting cellular and molecular drivers of head and neck squamous cell carcinoma: Current options and emerging perspectives. Cancer Metastasis Rev 35: 413-426, 2016.

4. Zanoni DK, Montero PH, Migliacci JC, Shah JP, Wong RJ, Ganly I and Patel SG: Survival outcomes after treatment of cancer of the oral cavity (1985-2015). Oral Oncol 90: 115-121, 2019.

5. Dobreva G, Dambacher J and Grosschedl R: SUMO modification of a novel MAR-binding protein, SATB2, modulates immunoglobulin mu gene expression. Genes Dev 17: 3048-3061, 2003.

6. Xu HY, Fang W, Huang ZW, Lu JC, Wang YQ, Tang QL, Song GH, Kang Y, Zhu XJ, Zou CY, et al: Metformin reduces SATB2-mediated osteosarcoma stem cell-like phenotype and tumor growth via inhibition of $\mathrm{N}$-cadherin/NF- $\kappa \mathrm{B}$ signaling. Eur Rev Med Pharmacol Sci 21: 4516-4528, 2017.

7. $\mathrm{Yu}$ W, Ma Y, Shankar S and Srivastava RK: SATB2/ $\beta$-catenin/TCF-LEF pathway induces cellular transformation by generating cancer stem cells in colorectal cancer. Sci Rep 7: $10939,2017$.

8. Joseph JP, Harishankar MK, Pillai AA and Devi A: Hypoxia induced EMT: A review on the mechanism of tumor progression and metastasis in OSCC. Oral Oncol 80: 23-32, 2018.

9. Pérez-Sayáns M, Suárez-Peñaranda JM, Pilar GD, Barros-Angueira F, Gándara-Rey JM and García-García A: Hypoxia-inducible factors in OSCC. Cancer Lett 313: 1-8, 2011.

10. Daskalaki I, Gkikas I and Tavernarakis N: Hypoxia and selective autophagy in cancer development and therapy. Front Cell Dev Biol 6: 104, 2018.

11. Vadde R, Vemula S, Jinka R, Merchant N, Bramhachari PV and Nagaraju GP: Role of hypoxia-inducible factors (HIF) in the maintenance of stemness and malignancy of colorectal cancer. Crit Rev Oncol Hematol 113: 22-27, 2017.

12. Dong W, Zhang P, Fu Y, Ge J, Cheng J, Yuan H and Jiang H: Roles of SATB2 in site-specific stemness, autophagy and senescence of bone marrow mesenchymal stem cells. J Cell Physiol 230: 680-690, 2015.

13. Mizushima N: Methods for monitoring autophagy. Int J Biochem Cell Biol 36: 2491-2502, 2004.

14. Yu W, Ma Y, Shankar S and Srivastava RK: Role of SATB2 in human pancreatic cancer: Implications in transformation and a promising biomarker. Oncotarget 7: 57783-57797, 2016.

15. Fukuhara M, Agnarsdóttir M, Edqvist PH, Coter A and Ponten F: SATB2 is expressed in Merkel cell carcinoma. Arch Dermatol Res 308: 449-454, 2016.

16. Chung J, Lau J, Cheng LS, Grant RI, Robinson F, Ketela T, Reis PP, Roche O, Kamel-Reid S, Moffat J, et al: SATB2 augments $\triangle \mathrm{Np} 63 \alpha$ in head and neck squamous cell carcinoma. EMBO Rep 11: 777-783, 2010.

17. Song S, Tan J, Miao Y, Li M and Zhang Q: Crosstalk of autophagy and apoptosis: Involvement of the dual role of autophagy under ER stress. J Cell Physiol 232: 2977-2984, 2017.

18. Thorburn A: Apoptosis and autophagy: Regulatory connections between two supposedly different processes. Apoptosis 13: 1-9, 2008.

19. Park BS, Choi NE, Lee JH, Kang HM, Yu SB, Kim HJ, Kang HK and Kim IR: Crosstalk between fisetin-induced apoptosis and autophagy in human oral squamous cell carcinoma. J Cancer 10: 138-146, 2019.

20. Wang X, Liu W, Wang P and Li S: RNA interference of long noncoding RNA HOTAIR suppresses autophagy and promotes apoptosis and sensitivity to cisplatin in oral squamous cell carcinoma. J Oral Pathol Med 47: 930-937, 2018.

21. Sophia J, Kowshik J, Dwivedi A, Bhutia SK, Manavathi B, Mishra R and Nagini S: Nimbolide, a neem limonoid inhibits cytoprotective autophagy to activate apoptosis via modulation of the PI3K/Akt/GSK-3 $\beta$ signalling pathway in oral cancer. Cell Death Dis 9: 1087, 2018. 
22. Lee $\mathrm{S}$ and Schmitt CA: The dynamic nature of senescence in cancer. Nat Cell Biol 21: 94-101, 2019.

23. Lathia JD and Liu H: Overview of cancer stem cells and stemness for community oncologists. Target Oncol 12: 387-399, 2017.

24. Li Y, Liu YH, Hu YY, Chen L and Li JM: Special AT-rich sequence-binding protein 2 acts as a negative regulator of stemness in colorectal cancer cells. World J Gastroenterol 22: 8528-8539, 2016.

25. Zhu LF, Hu Y, Yang CC, Xu XH, Ning TY, Wang ZL, Ye JH and Liu LK: Snail overexpression induces an epithelial to mesenchymal transition and cancer stem cell-like properties in SCC9 cells. Lab Invest 92: 744-752, 2012.
26. Mills CC, Kolb EA and Sampson VB: Development of chemotherapy with cell-cycle inhibitors for adult and pediatric cancer therapy. Cancer Res 78: 320-325, 2018.

27. Molinari M: Cell cycle checkpoints and their inactivation in human cancer. Cell Prolif 33: 261-274, 2000.

(i) (9) This work is licensed under a Creative Commons Attribution-NonCommercial-NoDerivatives 4.0 International (CC BY-NC-ND 4.0) License. 